\title{
Féeries
}

Études sur le conte merveilleuX, XVII $-\mathrm{XIX}{ }^{\mathrm{e}}$ siècle

\section{Les fables, les contes et la Fable chez La Fontaine : le secret du livre XII}

Fables, Tales and "Fable" in La Fontaine: the Secret of the Book XII

\section{Patrick Dandrey}

\section{(2) OpenEdition}

12 Journals

\section{Édition électronique}

URL : http://journals.openedition.org/feeries/755

DOI : $10.4000 /$ feeries. 755

ISSN : 1957-7753

\section{Éditeur}

UGA Éditions/Université Grenoble Alpes

\section{Édition imprimée}

Date de publication : 31 octobre 2010

Pagination : 45-74

ISBN : 978-2-84310-182-3

ISSN : 1766-2842

\section{Référence électronique}

Patrick Dandrey, «Les fables, les contes et la Fable chez La Fontaine : le secret du livre XII », Féeries [En ligne], 7| 2010, mis en ligne le 31 juillet 2011, consulté le 08 septembre 2020. URL : http:// journals.openedition.org/feeries/755; DOI : https://doi.org/10.4000/feeries.755

\section{(c) Féeries}




\author{
Patrick Dandrey \\ Université Paris-Sorbonne \\ Président de la Société des amis de Jean de La Fontaine
}

\title{
LES FABLES, LES CONTES ET LA FABLE CHEZ LA FONTAINE : LE SECRET DU LIVRE XII
}

$L$

'EUVRE DE LA FONTAINE semble avoir été composée pour servir d'exemple et de support d'analyse au sujet traité par la livraison présente de Féeries. Fable et conte paraissent y faire couple et harmoniser leurs figures et leurs pas en une danse chorégraphiée comme au temps jadis : c'est-à-dire sans jamais mêler leurs corps et, quoique en composant leurs mouvements chacun sur ceux de l'autre en une souple alternance, sans confondre le registre du cavalier (le conte) et de la cavalière (la fable). Cette similitude dans la distinction, il semble même qu'y conspirent à la fois la chronologie et la poétique : la publication des Contes et des Fables jalonne de sa scansion chronologique la carrière de La Fontaine, pendant que leurs préfaces affinent progressivement la définition réciproque et contrastée de l'esprit et de l'art poétiques propres à chacun des deux genres. Reste que cette distribution bien réglée bute sur une exception : celle du livre XII des Fables, qui admet en son sein quelques contes. On peut passer par profits et pertes cette incongruité. On peut s'y arrêter aussi, pour savoir si elle fait indice - et dans ce cas, indice de quoi ${ }^{1}$ ?

Dessinée à large arpentage, la carrière de La Fontaine semble attester une répartition bien ordonnée entre ses deux inspirations de fabuliste et de conteur. Jusqu'en I658, on ne sait trop ce qu'il fit, sinon se former en écolier

I. Sur le livre XII des Fables, on verra notamment P. A. Wadsworth, "Le douzième livre des Fables», Cahiers de l'Association internationale des études françaises, n 26, 1974, p. I03-1I5. J. Grimm, "Le livre XII des Fables: somme d'une vie, somme d'un siècle?", Le Fablier, n I, I989 (La Fontaine et la tradition européenne de la fable. I - La fable d'Esope à La Fontaine. Actes de Château-Thierry, I6-I8 juin I989), p. 63-69. Id., "Malgré Jupiter même et le temps orageux". Pour une réévaluation du livre XII des Fables", Euvres et critiques, XVI, 2, 199I, p. 57-69 (repris dans Le Pouvoir des fables. Etudes lafontainiennes I, Tübingen, Günter Narr, coll. "Biblio I7», 1994, p. I40-I50 et I6I-I72). M.-O. Sweetser, "Les leçons du livre XII", Parcours lafontainien. D’Adonis au livre XII des Fables, Tübingen, Günter Narr, coll. «Biblio I7», 2004, p. 247-26r. 
consciencieux à la poésie : la grande d'abord, hérö̈que et guindée, puis sa cadette, assouplie de plus de naturel et de quelques afféteries et autres pointes choisies. Ne nous est guère restée de ce temps qu'une comédie imitée de Térence, L'Eunuque, imprimée et diffusée dans l'indifférence générale, semble-t-il, en I6542. Le nom de Térence vaut pour signature d'urbanité souriante, d'imitation humaniste des Anciens et de conformisme esthétique. Armé de quoi le "garçon de Belles Lettres» (comme le nomme alors son aîné et ami Tallemant des Réaux ${ }^{3}$ ) se place en 1658 dans le sillage puis sous le patronage du surintendant Nicolas Fouquet et de son milieu intellectuel et esthétique gouverné par Paul Pellisson, oracle des élégances poétiques. L'Adonis qui sert à La Fontaine de billet d'entrée auprès de son bientôt protecteur consonne de l'harmonie et de la délicatesse propres à ce goût qu'on qualifie de "galant" pour en désigner l'élégance éclairée par le sourire de la connivence et du badinage ${ }^{4}$. Y règne la demi-teinte et le demi-mot : on y joue la naïveté en restaurant les formes oubliées du Moyen Âge et de la première Renaissance marotique, en modulant les tonalités ambiguës des genres et des tons mêlés, burlesque tempéré ou gaillardise émoussée et nimbée dans l'allusion à peine appuyée. Ce pouvait être à La Fontaine une excellente école pour mûrir d'une part ce que serait le ton des contes italiens ou gaulois qu'il naturaliserait en exercices de style faussement ingénu et délicatement égrillard, et pour distiller d'autre part la délicate naïveté des fables ésopiques qu'il allait revêtir des ornements de la poésie afin de délecter des adultes jouant à se (faire) prendre pour des enfants. Mais l'esthétique de ce temps, jusqu'à la chute du surintendant en I66I, et même un peu au-delà, jusque durant la campagne menée par ses fidèles lors de son procès, demeure chez La Fontaine celle de la galanterie ornée évoluant au mieux de son inspiration vers l'équilibre instable et inquiet d'une médiocrité dorée toujours en alerte.

Démuni de protecteur et de commande par la chute de son mécène, le poète peut avoir tiré de son expérience chez les galants l'idée de rimer les nouvelles de l'Arioste et les contes de Boccace en un mixte mi-souriant mi-incisif dont un premier bouquet va se constituer peu à peu, par livraisons successives de recueils progressivement augmentés : entre fin I664 et début 1665 pour une Première partie, et en 1666 pour la Deuxième partie,

2. L'Eunuque. Comédie, Paris, A. Courbé, 1654. J. de La Fontaine, CEuvres diverses, éd. critique de P. Clarac, Paris, Gallimard, coll. «Bibliothèque de la Pléiade», (1948) 1958, devenu CEuvres complètes II (ci-après référencé : OC II), p. 263-348.

3. P. Tallemant des Réaux, Historiettes, éd. critique d'A. Adam, Paris, Gallimard, coll. «Bibliothèque de la Pléiade", I960, 2 vol., I, p. 39I-392.

4. Adonis, OC II, p. 3-19. 
cependant qu'en I667 paraissent trois nouveaux contes qui enrichiront une réédition de cette Deuxième partie en 1669'. Entre-temps, les Fables ont été réunies en un volume illustré qui paraît au printemps I668. La dernière, en forme d'Épilogue, se termine par l'annonce du récit prosimétrique des Amours de Psyché et de Cupidon qui, achevé sans doute durant la période de composition éditoriale des Fables choisies et mises en vers, parait, lui, au début de $1669^{6}$. En I67I sortent presque simultanément une Troisième partie des contes et huit fables nouvelles réunies à des pièces de la période Fouquet ${ }^{7}$. Puis, en I674, de Nouveaux contes dont la gaze censée voiler les licences morales et les verdeurs grivoises n'empêche pas que tant d'audace (im)morale ne frappe d'un coup fatal sinon la carrière académique du poète, du moins la veine la plus hardie du conteur : le 5 avril I675, une sentence de police ordonne que l'on se saisisse du livre et que la vente en soit interdite ${ }^{8}$.

Deux ans plus tard, c'est donc pour des fables nouvelles que La Fontaine demande très logiquement un privilège. Les cinq nouveaux livres qu'il ajoute aux six de I668 paraitront en I678 (pour les deux premiers) et I679 (pour les trois suivants) 9 . Comme pourtant ses escapades dans divers genres, traductions, opéras, poésie dramatique ou scientifique, ne l'ont pas dissuadé de continuer à jalonner sa carrière de fables et même de contes dont il publie parfois isolément certains, dans le Mercure galant notamment, et d'autres en bouquets transitoires, comme les Ouvrages de prose et de poésie des sieurs de Maucroix et de La Fontaine en 1685, il finit par lier le tout en une ultime gerbe qui forme en 1694 le douzième et dernier livre des Fables choisies $^{10}$. Surprenante plasticité de cette partie rapportée, voici que s'ajoutent aux vingt-trois premières fables qu'il contient

5. Contes et nouvelles en vers de M. de La Fontaine, Paris, C. Barbin, 1665. Deuxième partie des Contes et nouvelles en vers de M. de La Fontaine, Paris, L. Billaine ou C. Barbin, 1666 (par erreur 1646). Contes et nouvelles en vers de M. de La Fontaine, Paris, C. Barbin, I669. J. de La Fontaine, Euvres diverses, ouvr. cité. J. de La Fontaine, Euvres complètes. I. Fables et contes, éd. critique de J.-P. Collinet, Paris, Gallimard, coll. «Bibliothèque de la Pléiade», I99I (ci-après référencé : OC I), p. 549-698.

6. Fables choisies et mises en vers, Paris, C. Barbin ou D. Thierry, I668, OC I, p. I-24I. Les Amours de Psyché et de Cupidon (joint la première édition d'Adonis), Paris, C. Barbin, I669, OC II, p. I2I-259.

7. Contes et nouvelles en vers de M. de La Fontaine. Troisième partie, Paris, C. Barbin et D. Thierry, I67I, OC II, p. 699-808. Fables nouvelles et autres poésies de M. de La Fontaine, Paris, C. Barbin, I67I, OC II, p. 597-608 et 950-954.

8. Nouveaux contes de M. de La Fontaine, Mons, G. Migeon, I674, OC I, p. 809-893.

9. Fables choisies et mises en vers, Paris, D. Thierry, I678, 3 vol., t. III (livres VII et VIII), OC I, p. 243-34I. En I679 paraît un t. IV (livres IX à XI), OC I, p. 343-445.

Io. Ouvrages de prose et de poésie des sieurs de Maucroix et de La Fontaine, Paris, C. Barbin, I685, OC II, p. 650-655 et 991-995. Le Mercure galant, décembre I690, février et mars I69I, décembre I692. 
et avant la dernière qui sert de conclusion générale aux volumes (Le Juge arbitre, le Solitaire et l'Hospitalier) un groupe de cinq pièces qui sembleraient n'avoir que faire là, si les dix fables qui les précèdent n'avaient déjà vogué de conserve avec elles dans le recueil déjà cité des Ouvrages de prose et de poésie. Il s'agit, respectivement, d'une idylle : Daphnis et Alcimadure. Imitation de Théocrite; d'un conte mythologique et édifiant: Philémon et Baucis. Sujet tiré des "Métamorphoses» d'Ovide; d'un conte à l'antique assez leste : La Matrone d'Éphèse; d'une nouvelle non moins plaisamment misogyne : Belphégor. Nouvelle tirée de Machiavel; et d'une autre métamorphose ovidienne: Les Filles de Minée. Sujet tiré des "Métamorphoses» d'Ovide. Ces pièces procédant des deux inspirations - conteuse et ésopique - qu'on aurait attendu plus étanches se présentaient d'ailleurs dans un ordre à peu près similaire à celui qu' elles occupaient dans le recueil de 1685 et à quelques variantes de texte près.

Faut-il en déduire que ces contes et nouvelles de facture diverse et d'orthodoxie morale non moins diverse sont dans l'esprit du poète devenus semblables et assimilables à des fables? Ou que les fables, en leur dernier décours et au terme de tant d'évolutions plastiques qui les ont presque dénaturées, ne peuvent plus se définir que par leur plus petit commun dénominateur, celui du conte en vers? Ou encore que, trop spécifique pour faire genre à lui seul, l'apologue qui ne fut jamais qu'une province de l'empire narratif avoue ainsi in extremis son inclusion partielle et non réciproque au genre du conte en vers, en lieu et place du parallélisme et de l'étanchéité génériques que la pratique de La Fontaine et le cheminement de son œuvre nous ont habitués à recevoir pour évidence? Ou si, tout à l'inverse, l'indétermination du conte ou de la nouvelle en vers, genre fuyant, moderne, trop souple pour être régulé, reconnaissait ici la suzeraineté de l'autre genre, celui que La Fontaine avait fini par hisser au statut inouï de miroir microcosmique de la littérature tout entière dont l'apologue se sera peu à peu entendu à imiter en miniature toutes les compositions : ainsi, de même qu'il est des fables qui s'avouent comédies, épopées, élégies, panégyriques ou apologies en miniature - ainsi, et tout de même, du conte, dont l'apologue phagocyterait à son tour le génie par cette annexion ultime et ostensible?

Quoi qu'il en soit, n'était-ce pas pour La Fontaine démentir discrètement et paradoxalement trente ans non seulement de pratique, mais d'efforts théoriques pour distinguer les deux modes majeurs de son ins-

Fables choisies, mises en vers par M. de La Fontaine et par lui revues et augmentées, Paris, C. Barbin, I692 (4 vol.) - I694 (I vol., formant la $5^{\mathrm{e}}$ partie qui contient le livre XII actuel). 
piration? Divergence d'origine, d'abord. Celle des contes en vers (dédoublés, rappelons-le, en contes proprement dits et en «nouvelles» dont Joconde est l'archétype) procède de voisinage, un voisinage dans le temps, celui de la Renaissance, et dans l'espace, celui de l'Italie : Boccace pour les contes, l'Arioste pour les nouvelles. L'origine des fables (elles aussi divisées en un massif ésopique, premier, bientôt complété d'un autre emprunté à la tradition dite de Pilpay) procède dans les deux cas d'horizons bien plus lointains : distance dans le temps, celui de l'Antiquité gréco-latine, ou dans l'espace, celui de l'Orient lointain. L'adaptation de ces sources opère, elle aussi, de manière divergente : si dans les deux cas l'on traduit et versifie, les contes sont pour la plupart tournés en décasyllabes réguliers (davantage dans les débuts de La Fontaine, moins vers la fin de sa carrière de conteur), alors que les fables appellent des mètres irréguliers associant leur disparate parfois jusqu'au vertige - un alexandrin peut y faire couple avec un vers de trois syllabes, comme dans les vers 28 et 29 des Animaux malades de la peste ${ }^{\mathrm{II}}$. Quant aux nouvelles, l'irrégularité du mètre qui semble y calquer la fluidité de la prose narrative se rapproche bien davantage, quelque paradoxe qu'il y ait à le dire, du rythme des contes tirés de Boccace que de la sophistication avec laquelle est ciselé le très bref récit des apologues. Brièveté ornée, certes, et jouant à tanguer entre l'ellipse et la glose, peutêtre; reste que la fable, quels que soient les corollaires et les incises de sa narration qui s'en enrichit et s'y disperse, ramasse toujours plus ou moins son génie en un mince événement autour duquel elle se concentre : l'unité de la cellule narrative est son principe. Le conte et la nouvelle, eux, déroulent plus volontiers une narration linéaire à épisodes : même dans les cas où ils réduisent en quelques vers une épigramme, leur principe demeure celui de la glose, dont le génie est de développer, quand celui de la fable est de schématiser. Le conte déroule, fût-ce avec force méandres; la fable concentre, fût-ce avec quelques gloses.

Quant à ce qu'ils content l'un et l'autre, la divergence semble encore plus éclatante : l'apologue recourt à la transposition, sans craindre d'user du registre animalier, végétal, voire minéral ou abstrait, alors que les récits du conte et la nouvelle n'excèdent guère l'ordre humain; ou, s'ils recourent à la mythologie, c'est à une mythologie pour rire, où les diables sont de bons bougres et les dieux de simples gens, à tu et à toi avec les mortels, sauf qu'ils se montrent un peu plus puissants que le commun. Tels ceux d'Homère, en somme. Au demeurant, cette référence à l'humanité

II. "Même il m’est arrivé quelquefois de manger / Le Berger ", Fables, VII, I, OC I, p. 250. Sur le mètre lafontainien, voir G. Peureux, La Fabrique du vers, Paris, Seuil, coll. «Poétique», 2009. 
n'est guère qu'un prétexte pour les contes et nouvelles, puisque, avoue le conteur, "ce n'est ni le vrai ni le vraisemblable qui font la beauté et la grâce de ces choses-ci; c'est seulement la manière de les conter ${ }^{12}{ }^{\prime}$. Le fabuliste au contraire rappelle que "conter pour conter [lui] semble peu d'affaire ${ }^{\mathrm{I3}}$. Car, instructif et édifiant, l'apologue prétend à enseigner, à enseigner notamment et surtout les enfants, même si c'est par convention qu'on leur destine ces bijoux subtils plus propres à parer des adultes. Pour ce faire, la fable laisse place, en son début ou sa fin, à un décrochement qui rompt conventionnellement l'illusion narrative pour tirer la morale de l'histoire. La nouvelle et même le conte tels que les pratique La Fontaine ne s'embarrassent pas d'instruire mais s'efforcent à plaire, et à plaire aux adultes, singulièrement aux hommes que n'effraie pas la gaillardise, tout en n'interdisant pas aux dames de s'y délecter discrètement. De même, mais dans son ordre, la puérilité de la fable tâche de viser en chaque adulte l'enfant qui y sommeille et à jouer sur la connivence entre ces registres : gaillardise faussement grivoise et naïveté faussement puérile décalent ainsi l'effet concerté de la mise en œuvre par rapport à l'intention convenue de chacun des deux genres. Enfin, s’ils délivrent une leçon, les contes n’architecturent pas leur narration sur le principe du dialogue codifié entre récit et moralité, que la Préface des Fables choisies assimile au contraire à l'association du corps et de l'âme dans l'ordre humain ou, métaphoriquement, dans l'art de l'emblème ${ }^{\mathrm{I} 4}$.

En deux mots donc et qui résument cette différence, si même ils ne la fondent, la fable se distingue structuralement du conte par sa double articulation; et le reste s'en ensuit. D'un côté, elle projette la naturehumaine-universelle (du moins en juge-t-elle ainsi) dans la singularité décalée, didactique et particularisée d'un ordre de réalité schématique, coloré, transposé : celui du monde animal, végétal, voire matériel ou symbolique et, lorsqu'il est humain, réduit à des santons absorbés par un seul attribut de l'humaine condition (profession, rang, trait physique ou de mœurs). D’un autre côté, elle projette cette transposition paradigmatique sur l'axe syntagmatique en enchaînant la dénotation à la connotation, le décryptage au chiffrage, bref la moralité au récit, l'âme au corps, et en invitant le lecteur à les superposer, à fusionner leurs leçons. La poétique du conte, à côté, fait figure de parent pauvre. Restaurons une vieille distinction grammaticale pour le dire en une formule : comme en langue le

I2. Préface de la Première partie des Contes et nouvelles en vers, OC I, p. 557.

13. Le Pâtre et le Lion, Fables, VI, I, v. 6, OC I, p. 209.

I4. Préface des Fables choisies (I668), OC I, p. 9. 
féminin est réputé se distinguer du masculin non par la sexualité de ce qu'il nomme, mais par la marque du -e final (ou caduc) qu'il ajoute à certains vocables, ce qui permet de définir structuralement leur opposition comme celle du marqué au non-marqué, de même la fable pourrait être définie comme le genre "marqué» par rapport au conte "non marqué». D’où les englobements décalés et non réciproques qu'on a rappelés : la fable est enveloppée dans le genre du conte comme une de ses modalités mineures, le conte dans le genre de la fable comme un des ses constituants formels. La divergence ne relève plus ici du parallèle; elle relève du décalage. Et ce n'est d'ailleurs que le premier décalage d'une plus longue liste. En effet, la double articulation que l'on vient de dire, dans l'esprit entre métaphore et référent, dans l'espace entre récit et moralité, implique aussi un double décalage dans le parallèle entre les deux genres.

D'abord, leurs divergences se décalent de l'esthétique à la générique. Le traitement qu'applique La Fontaine aux deux genres, c'est-à-dire la versification ${ }^{15}$, suggère une intention et une pratique esthétiques similaires : on ne saurait guère tirer conséquence, sinon de manière anecdotique et pratique, de la divergence que l'on a dite plus haut dans l'art de narrer en vers du fabuliste et du conteur. Rien là de radicalement, de fondamentalement divergent. Radicale, en revanche, fondamentale et fondatrice, la dénivellation structurale : la fable enfouit ses soubassements dans un tréfonds de sagesse populaire qu'elle renouvelle et vivifie par un effet de transfert, un mécanisme métaphorique au sens le plus étymologique, une transposition de la réalité humaine dans un autre univers, celui de la zoologie étant devenu l'emblème du genre. En quoi elle consonne avec la poésie, si l'on veut bien mettre au principe de celle-ci un exercice de transposition dans le langage et dans l'image à des fins d'enchantement de l'âme et d'illumination de l'esprit par l'effet déstabilisant d'une superposition décalée de représentations. En promouvant en poésie l'apologue, La Fontaine ne faisait qu'accomplir son principe : celui de la métaphore. Le conte, lui, ne s'enracine que dans sa tradition, à peine régulée, sans ambition de sagesse qu'anecdotique ou surajoutée, mais sans rien d'intrinsèque à sa définition.

Il n'est pas fortuit qu'une confusion de langage nomme Fable le conte, le mythos, dès lors qu'il est envisagé dans l'optique du déchiffrement de son plus haut Sens. Le conte, chez La Fontaine, c'est une fable sans Fable, un apologue sans âme, un récit sans morale - même lorsqu'il en a une, implicite ou délivrée. Certes, il est bien vrai et il faut rappeler que, sous

15. Laquelle doit s'entendre, on le sait bien, comme le simple indice de leur projection en poésie, qui constitue pour le coup un phénomène de bien plus grande conséquence. 
l'influence peut-être de son métier de fabuliste en cours d'apprentissage, La Fontaine qui avait servi ses premiers contes tout de go et de but en blanc commença à faire précéder certains récits de la Deuxième partie des contes et nouvelles par une réflexion générale, assumée, glosant une observation de caractères ou de mœurs, éventuellement une maxime de conduite universelle, ou simplement une intrusion du narrateur commentant ce qu'il va dérouler ${ }^{16}$ : le récit qui fait l'essentiel du poème prend alors explicitement valeur $\mathrm{d}^{\prime}$ " exemple ${ }^{17}$ » par rapport à cette entrée en matière. Mais justement : il ne s'agit que d'une entrée en matière. Et personne ne songerait à attribuer le statut d'âme à ces bavardages liminaires dont le conte formant tout au plus le corps recevrait sa vie et son sens. Réciproquement, si la promotion de certaines fables en poésie a empêché que leur moralité soit explicitée, elle n'en subsiste pas moins, implicite et évidente, comme moteur, comme âme du récit qu'elle continue de subsumer de son aura, d'envelopper de son intention, d'éclairer de son déchiffrement.

Ensuite et par suite, seconde divergence imputable à la double articulation de la fable qu'ignore totalement le conte, ce n'est pas que leur accès au sens diverge, c'est que la première chiffre et dénote sa signification par l'ostentation du phénomène de transposition qui la fonde, tandis que l'autre accède au sens de manière directe et immédiate, sans principe de dénivellation. Autre manière de le dire : la fable est par essence allégorique, le conte sinon tautégorique, du moins seulement didactique et paradigmatique, même lorsqu'il prétend illustrer quelque maxime de conduite humaine et plus précisément masculine... ou féminine, bien sûr. Car le passage du particulier au général y procède de déduction et non de transposition, et le passage du général au particulier d'illustration et non de codage.

De quoi dès lors la «bigarrure» du XII livre fait-elle indice? Violeraitelle, plus grave encore que la distinction esthétique entre les deux genres, l'esprit même de leur différence? Pour répondre à cette question autrement qu'en termes de laisser-aller ou de pis-aller, pour mesurer comment cette tardive coalescence a pu se produire, encore faut-il revenir sur la présentation trop rapide et superficielle que nous en avons faite à l'instant et entrer dans plus de détail sur la genèse et la courbe de l'invention poétique de La Fontaine taillée dans ces deux veines.

On ne sait à dire vrai comment il vint à la fable et quand il le fit, mais quelques indices permettent de remonter cette inspiration assez tôt avant

I6. Cela commence avec L'Oraison de saint Julien, puis La Servante justifiée, etc., OC I, p. 628 et suivantes.

17. Le Calendrier des vieillards, v. 17, À femme avare galant escroc, v. Io, etc., OC I, p. 650, $657 \ldots$ 
la parution en 1668 de son premier recueil de quelque cent vingt-quatre Fables choisies et mises en vers. La présence de dix apologues ésopiques dans le recueil manuscrit confectionné par Valentin Conrart, qui ne les eût pas reproduites et en une version souvent moins achevée que celle de l'édition originale si celle-ci avait déjà vu le jour, permet de supposer que ces textes circulaient, épars et encore peu nombreux, dès avant et peut-être longtemps avant leur multiplication et leur liage en une gerbe poétique à peu près achevée en juin $\mathrm{I} 667$, quand le libraire Barbin partage avec son collègue Thierry le privilège qu'il a demandé pour leur impres$\operatorname{sion}^{18}$. Confirme cette supposition l'existence d'un autre recueil manuscrit conservé par la bibliothèque Sainte-Geneviève, et ajoutant neuf autres pièces à six de celles qu'a transcrites Conrart ${ }^{19}$. Toutes étaient appelées à se retrouver dans le recueil de 1668, sauf deux : La Poule et le Renard, du manuscrit Sainte-Geneviève, d'authenticité incertaine; et Le Renard et l'Écureuil, du manuscrit Conrart, dont l'application patente au conflit entre le surintendant Fouquet, protecteur de La Fontaine, et son dénonciateur Colbert suggère de remonter la gestation aux années du procès qui suivit la chute du premier (entre septembre I66I et décembre I664). Peut-on attribuer à La Fontaine cet apologue absent de son recueil? Plus sûrement en tout cas que l'autre, depuis la récente découverte d'un écureuil caché dans les frondaisons d'une gravure de Henri Cause d'après Chauveau, et peut-être même prise directement à un modèle de celui-ci ${ }^{20}$. La gravure orne une contrefaçon hollandaise du second recueil des Fables, celui de 1678. Comme si, à un moment indéterminé de la gestation de ses apologues mais certainement bien antérieur à l'époque de ce remploi, La Fontaine avait eu l'intention d'y glisser celle de l'Écureuil et du Renard. Ce qui donne bien du poids à l'hypothèse d'une rédaction des premières fables dans le cadre et aux fins de la campagne d'opinion menée durant le procès de Fouquet par ses partisans, au premier nombre desquels l'Ode au

18. Valentin Conrart, ms. 5418 de la Bibliothèque de l'Arsenal, t. XI, fos 533-539. S'y trouvent Le Loup et l'Agneau, Le Corbeau et le Renard, Les Grenouilles qui demandent un roi, Deux mulets, Le Renard et l'Écureuil, La Génisse, la Chèvre et la Brebis en société avec le Lion, La Grenouille qui veut ressembler au boeuf, Le Lion accablé de vieillesse, Le Rat de ville et le Rat des champs, La Mort et le Malheureux. Voir B. Donné, «La Fontaine et l'invention des Fables», Le Fablier, n 19, 2008, p. $7 \mathrm{I}-84$.

19. Ms. 2445 de la Bibliothèque Sainte-Geneviève, fos $176-186$. Les fables ajoutées sont : La Poule et le Renard, Le Vieillard et ses Enfants, Le Paon se plaignant à Junon, Le Geai qui s'est paré des plumes du paon, Le Vieillard et l'Âne, La Poule aux oufs d'or, Le Cheval s'étant voulu venger du cerf, Le Conseil tenu par les rats, Le Loup et le Renard plaidant devant le singe.

20. Découverte due à B. Donné, ouvr. cité, dont nous paraphrasons en la schématisant la démonstration à la fois suggestive, complète et virtuose. 
Roi et l'Élégie pour M. F. (dite aux nymphes de Vaux) placent sans conteste La Fontaine ${ }^{21}$. Voici le projet de rimer les fables ésopiques remonté jusqu'au début de la décennie i660 au plus tôt, à son milieu au plus tard.

Poussons un peu plus haut encore, avec précaution : durant les années où il est l'obligé et bientôt le pensionné de Fouquet, depuis la mi-cinquantehuit, La Fontaine a entrepris une évocation en vers et sous forme de songe des merveilles de Vaux dont le surintendant faisait édifier le château et réaliser les jardins. Ce Songe de Vaux inachevé offre notamment deux pièces en vers inégaux, l'"Aventure d'un saumon et d'un esturgeon» et les "Aventures du cygne" dont "Sylvie honora de sa présence les dernières chansons ${ }^{22}$, pièces qu'on n'ose dire ésopiques, mais du moins animalières et piquantes, dans le goût et le tour "galants» que le recueil de I668 allait donner aux animaleries effectivement ésopiques de Phèdre et du fonds d'apologues antiques connus sous le nom légendaire d'Ésope ${ }^{23}$. Indice, vague indice, d'un intérêt et d'une pratique poétiques qui pouvait mettre, allait mettre, avait peut-être déjà mis La Fontaine sur la piste des fables ésopiques. Plus faible indice encore, on tient d'ordinaire deux fables en alexandrins réguliers, Le Meunier, son Fils et l'Âne et Le Vieillard et ses Enfants pour l'expression des premières armes du poète dans le genre de la fable ésopique. La première, d'inspiration italienne moderne, parvenue par l'intermédiaire de Malherbe jusqu'à La Fontaine, aurait été composée par lui en I647, selon le témoignage tardif de Brossette (1716), à propos des atermoiements de François Maucroix, l'ami de toute une vie à qui elle est dédiée, lequel hésitait entre le barreau, l'Église et le mariage ${ }^{24}$. Rien ne dit que ce témoignage soit recevable ni que l'écriture de la fable date de cette époque, mais rien ne dit non plus le contraire. La coïncidence du sujet de l'autre fable avec l'embarras des affaires de La Fontaine à la mort de son père en 1658 convainc moins.

En dépit de cette remontée fort haut dans la première inspiration du fabuliste, le premier recueil des Fables a été, comme on sait, devancé de quatre ans par la première livraison des Nouvelles en vers tirée[s] de Boccace et de l'Arioste dont le privilège pris en janvier I664 couvrait Joconde, dé-

2I. Ode au Roi [fin I662 ou début I663?], OC II, p. 530-532. Élégie pour M. F., anonyme, s. 1.n.d. [1662?], OC II, p. 528-530.

22. Le Songe de Vaux, III et IV, OC II, p. 97-104.

23. Pour une mise au point essentielle et définitive sur cet aspect de la question ésopique, voir A. Biscéré, «Les fables d'Ésope, une œuvre sans auteur?», Le Fablier, n²0, 2009, p. 9-36.

24. Le Meunier, son Fils et l'Âne. À M. D. M., Fables, III, I, OC I, p. I05-107. Brossette, Euvres de M. Boileau-Despréaux, avec des éclaircissements historiques donnés par lui-même, Genève, Fabri et Barrillot, I716, II, p. 324. La dédicace s’entendrait donc : À Monsieur de Maucroix. 
marquée d'un épisode du Roland furieux, qui est précédée dans l'édition parue en décembre 1664 et datée de 1665 par Le Cocu, battu et content, d'après Le Décaméron, et suivie d'une version de La Matrone d'Éphèse, en prose mêlée de (quelques) vers, due semble-t-il à La Valterie. Cette entrée discrète et composite dans le genre du conte annonçait la plus éclatante publication des treize Contes et nouvelles en vers un mois plus tard, en janvier I665, suivis d'une Deuxième partie, en janvier de l'année suivante, qui contient le même nombre de pièces, mais plus étoffées : celles-ci occupent soixante-dix pages de plus que n'en occupaient celles-là. Une préface, anticipant celle des Fables l'année suivante, complète la théorie du genre qu'esquissait celle du premier volume de I664-I $665^{25}$. Le tout achevait un massif d'ouvrages qu'apparemment le conteur estimait définitivement clos. Place était faite par l'achèvement des Contes, dès la fin I665, à la composition et à la réunion des Fables qui paraitraient, elles, en mars I668. Après quoi, la messe semblait dite et sa double carrière de conteur et de fabuliste s'achever, dans l'esprit du moins de La Fontaine.

C'était compter sans la fécondité de sa veine narrative et morale. Beaucoup de ce qu'il a composé avant I664, et bien plus encore, si l'on veut bien se souvenir qu'il a détruit ou recyclé toute sa première production antérieure à Adonis (1658), sauf L'Eunuque déjà cité, tout cela résonne de son talent de conteur : Joconde n'émerge pas d'un océan d'indifférence pour le conte en vers, mais d'une mer nourricière. Y surnagent la courte chanson du Curé de Bussière qui proposait d'échanger aux Allemands sa chambrière contre sa jument; peut-être le dizain contant l'historiette de sœur Claude (ou Jeanne) qu'on retrouve dans la Première partie des Contes et nouvelles; plus sûrement le récit des Amours de Mars et de Vénus, pastiche de mythologie dans le tour du burlesque tempéré inséré dans Le Songe de Vaux; et puis encore quelques rares pièces sauvées de la production éphémère composée pour la société castelthéodoricienne, d'esprit anti-monacal, gaillard et goliard ${ }^{26}$.

La césure que marque, au début 1669, la publication des Amours de Psyché et de Cupidon, ouvre exceptionnellement en prose quoique mêlée de vers, n'empêche pas la résurgence d'un flux qui a continué de couler

25. Nouvelles en vers tirée(s) de Boccace et de l'Arioste par M. de L. F., Paris, C. Barbin, 1665 (ach. d'impr. 1664). Préface de la Première partie des Contes et nouvelles, OC I, p. 557-557 et de la Deuxième partie, OC I, p. 603-605.

26. Le Curé de Bussières, OC II, p. 479. Le Conte de ${ }^{* * *}$ figurait déjà dans P. Richelet éd. (?), Les Plaisirs de la poésie galante, gaillarde et amoureuse (partie non paginée), de date incertaine mais antérieure à 1665. Les Amours de Mars et de Vénus. Fragment, dans Le Songe de Vaux, IX, OC II, p. II5-IIg. Et diverses petites pièces recueillies dans les OC II, p. 488-490. 
souterrainement. Trois contes qui, parus en I667 dans un Recueil (clandestin) contenant plusieurs discours libres et moraux et quelques nouvelles en vers non encore imprimées, ne figuraient pas dans la première édition collective des deux tomes de Contes et nouvelles sortis la même année, entrent en revanche dans leur réédition en I669, par-delà donc la publication du premier recueil de Fables, avec une version incomplète de $L a$ Coupe enchantée dont le texte inachevé avait été livré peu avant sans l'aveu de l'auteur par Jean Sambix, éditeur à Leyde ${ }^{27}$. C'était l'amorce pour une troisième partie des Contes et nouvelles où La Coupe enchantée reparaîtra dans sa version définitive : parue en I671, la nouvelle moisson comporte, outre quatorze nouveaux contes, la "comédie» de Clymène, génériquement et chronologiquement incertaine, le tout protégé par trois privilèges, celui des Contes, celui des Fables, celui des Amours de Psyché28. Les quatorze pièces que comprend le volume n'offrent d'ailleurs pas une grande unité générique ni formelle : longueur très diverse, de quinze à trois cents vers, genres incertains, de l'épigramme au dialogue dramatique, mètres différents, à quoi s'ajoutent des conditions de publication confuses et une édition négligée et fautive. L'unité de façade que suppose le titre du volume se délite en une troublante confusion.

D'autant plus troublante qu'au même moment, en mars I67I, paraît le volume non moins disparate des Fables nouvelles et autres poésies: il offre huit apologues inédits appelés à reparaître dans les recueils de 1678 et I679, mais aussi diverses pièces remontant au temps de Fouquet et mêlées à d'autres de provenance récente. Rien encore ici de chronologiquement ni génériquement unifié. On devine seulement que, durant ces années intermédiaires, le flux des fables continue de couler, aux résurgences que l'on observe, conservées jusqu'à nous par le hasard des allusions décryptées ou des feuilles volantes conservées : en I672, la petite histoire fournit

27. Les Cordeliers de Catalogne (devenu Les Frères de Catalogne), L'Ermite et Le Muet (devenu Mazet de Lamporechio) figuraient dans le Recueil contenant plusieurs discours libres et moraux et quelques nouvelles en vers non encore imprimées, Cologne, À la Sphère, I667, p. 48-68. Ils se retrouvent dans les Contes et nouvelles en vers de M. de La Fontaine, Paris, C. Barbin, I669 (OC I, p. 6126I8 et 687-698). Le prologue de La Coupe enchantée (v. I-78 de la version définitive) figurait, seul, dans le ms. de la Bibliothèque Saint-Geneviève déjà cité, fos $176-178$. Ce prologue suivi d'un fragment inachevé était paru dans l'édition non avouée des Contes et nouvelles en vers de M. de La Fontaine. Nouvelle édition revue et augmentée de plusieurs contes du même auteur et d'une dissertation sur la (sic) Joconde, Leyde, Jean Sambix le jeune, I669. La version définitive de la nouvelle, sans le fragment de l'édition Sambix, figure dans la Troisième partie... de i67I (OC I, p. 720-732).

28. On date volontiers Clymène du temps de Vaux, voire de I658 (OC II, p. 804-805). Voir J.-Ch. Darmon, Philosophies de la Fable. La Fontaine et la crise du lyrisme, Paris, PUF, coll. "Écriture», 2003, Première partie, p. I3-IOI. 
de matière Le Curé et le Mort, et la grande Le Soleil et les Grenouilles; en I674, une lettre de $\mathrm{M}^{\mathrm{me}}$ de Sévigné nous apprend que circule une version manuscrite de La Cour du Lion, à moins que ce ne soient Les Animaux malades de la peste, dont l'Arsenal possède une version manuscrite datée de la même année ${ }^{29}$. Quant à une copie manuscrite de Tircis et Amarante (d'authenticité et d'existence douteuses), on n'en dira rien puisqu'elle a disparu depuis qu'au XIX ${ }^{\mathrm{e}}$ siècle elle avait été mentionnée dans la collection Feuillet de Conches, d'autorité très discutée. Tout cela nous achemine pourtant vers le projet du nouveau recueil des fables. Mais non sans que survienne sur sa route le nouveau et dernier recueil des contes.

Ces Nouveaux contes, poursuivant sur la voie licencieuse des précédents, renchérissent sur eux de libertés et de grivoiseries. Sortis des presses sous une adresse éditoriale fictive, sans privilège ni permission ${ }^{30}$, ils semblent avoir eux aussi été préparés par des circulations de textes s'entrecroisant, voire s'entremêlant avec celle des fables destinées à entrer dans la constitution du nouveau recueil de I678-1679. Par exemple, Les Troqueurs sont déjà parus dans une plaquette volante signée M.D.L.F et sans date; mais une copie manuscrite qu'en offre le recueil Du Tralage porte celle de I672, quand La Fontaine composait Le Soleil et les Grenouilles, et peut-être aussi Le Curé et le Mort. D'autres versions d'autres contes ont sans doute existé, perdues depuis. En tout cas, il est clair que de I67I à I674 La Fontaine n'aura cessé de composer dans les deux genres. Sur le volume des Nouveaux contes, autant qu'on puisse le savoir, l'impression dominante sera que le poète est allé, sinon trop loin, aussi loin en tout cas que possible. Son élection à l'Académie va s'en trouver chahutée. Furetière, déchaîné contre la compagnie après en avoir été exclu pour avoir composé un dictionnaire concurrent du sien, ne manquera pas de rallumer cette braise en traitant

29. Le Curé et le Mort, Fables, VII, IO, OC I, p. 268-269 (incident réellement survenu, évoqué par $\mathrm{M}^{\mathrm{me}}$ de Sévigné, lettre du 26 février 1672). Le Soleil et les Grenouilles. Imitation de la fable latine, non recueillie, $O C I$, p. 54I-542 (durant la préparation de la guerre de Hollande). $\mathrm{M}^{\mathrm{me}}$ de Sévigné : "Voilà une fable des plus jolies; ne connaissez-vous personne qui soit aussi bon courtisan que le Renard?" (lettre du 22 mai I674), à propos de La Cour du Lion, VII, 6, ou des Animaux malades de la peste, VII, I, dont une version manuscrite se rencontre dans le recueil de J.-N. Du Tralage, Arsenal ms. 6544 .

30. Le nom de Gaspar Migeon à Mons semble cacher celui de Muletier à Reims ou de Bouchard à Châlons. Il faisait une allusion en forme de nasarde au patronyme de Gaspard Migeot qui avait imprimé en 1667 le Nouveau testament dit de Mons, dans la traduction désormais fameuse de Le Maître de Sacy. L'équivalence allusive entre nouveau testament et nouveaux contes obscènes pouvait parler aux fins connaisseurs de la librairie. La saisie des volumes par la police fut ordonnée par le lieutenant général La Reynie le 5 avril 1675. 
le conteur d' "Arétin mitigé ${ }^{31}$ ». La Fontaine allait-il pour autant renoncer aux contes? Aux contes de manière licencieuse et hardie, oui certes, de gré ou de force; mais au genre, non. En I682, il avait profité de la parution d'un poème scientifique à la gloire du Quinquina pour offrir une nouvelle gerbe d'œuvres mêlées (Poème du Quinquina et autres ouvrages en vers ${ }^{32}$ ) qui métamorphosait sa manière de conter, ou plutôt renouait avec celle de ses débuts : y figurent La Matrone d'Éphèse, en vers irréguliers comme les fables, et Belphégor, en décasyllabes de ton marotique, d'inspiration non moins digne de la gaieté qu’il avait conférée aux apologues ésopiques. Ce sont eux que l'on retrouvera dans le futur livre XII du recueil des Fables ${ }^{33}$.

Les cinq livres nouveaux de fables parus entre-temps, en I678 et I679, avaient renouvelé pour ne pas dire transfiguré le genre dans ses sources, ses formes et ses fins, en dissolvant bien des traits identifiants d'origine ésopique. Le public mondain avait prisé dans les fables de i668 le tour familier, primesautier et narquois, la sagesse faussement naïve et plaisamment acérée, les archaïsmes savoureux et la vigueur narrative de saynètes suggestives et vivement dialoguées. Un Ésope poétisé, en somme. Au lieu de quoi, comme l'expliquent et s'en justifient l'Avertissement initial et la longue dédicace en vers adressée "Â $\mathrm{M}^{\mathrm{me}}$ de Montespan ${ }^{34}$ », la matière des fables de 1678-1679 est en partie puisée à des sources encore inexploitées, pour bonne part orientales. Ce renouvellement de sujets a requis un infléchissement de leur manière, plus circonstanciée et ornée, plus subtile aussi, ductile, conteuse pour tout dire. Enfin, cette modification de goût leur a conféré une portée esthétique et morale moins simple et enjouée que naguère, plus nuancée et méditative, traversée d'ambitions éthiques et philosophiques de plus haut vol. En somme, d'un recueil à l'autre, la revendication proclamée d'un plaisir lettré s'est clairement substituée au

31. L'élection mouvementée de La Fontaine eut lieu le I5 novembre I683. Les factums de Furetière après l'annulation du privilège de son Dictionnaire et son éviction consécutive de l'Académie (le 22 janvier 1685) s'en prennent à quelques-uns des académiciens, dont La Fontaine, qui avaient voté sa déchéance. Ils ont été réédités par C. Asselineau, Recueil des factums d'Antoine Furetière, de l'Académie françoise, contre quelques-uns de cette Académie, suivi des preuves et pièces historiques données dans l'édition de I694, avec une introduction et des notes historiques et critiques, Paris, Poulet-Malassis et de Broise, I859, 2 vol. (repr. Farnborough, Gregg International, 197I). S'ensuivit un échange d'épigrammes avec La Fontaine (OC II, p. 646-647). Et une polémique à épisodes, durant laquelle Furetière compose notamment son Plan et dessein du poème allégorique et tragico-burlesque intitulé "Les couches de l'Académie» (1687). La formule d' "Arétin mitigé" était apparue dès janvier 1685 (Asselineau, I, p. I8I) et sera reprise dans le Troisième factum de décembre i686 (id., I, p. 300).

32. Poème du Quinquina et autres ouvrages en vers de M. de La Fontaine, Paris, D. Thierry et C. Barbin, I682, OC II, p. 62-77.

33. Fables, XII, 26 et 27, OC I, p. 508-52I.

34. OCI, p. $245-248$. 
prétexte pédagogique et didactique, en même temps qu'à la tradition ésopique succédait une inspiration plus variée, ouverte à tous les vents de la culture universelle et de la narration circonstanciée : le Livre des lumières dû au brahmane légendaire Pilpay, auquel certes le nouveau recueil doit beaucoup, n'aura constitué que le gisement le plus fécond parmi plusieurs autres.

La Fontaine n'en a pourtant pas encore fini avec la fable. Des pièces d'artifice isolées suivent encore ce bouquet. En I684, à l'Académie, pour la réception de Boileau, il lit Le Renard, le Loup et le Cheval, qui serait publiée l'année suivante, jointe à d'autres sans doute diffusées elles aussi isolément, dans les Ouvrages de prose et de poésie des sieurs de Maucroix et de La Fontaine, embryon du livre XII. En I689, pour le mariage du prince de Conti, il compose Le Milan, le Roi et le Chasseur, dont la version fixée dans ce même livre sera partiellement démentie par celle qu'en découvriront les Euvres posthumes de 1696. Entre la fin I690 et la fin I692, le Mercure galant publie successivement une première version des Compagnons d'Ulysse, puis sous l'anonymat Les deux Chèvres, et encore Du Thésauriseur et du Singe, enfin La Ligue des Rats, trop tard venue pour être insérée dans le douzième livre. Quant à celle qui deviendra la dernière fable du livre XII et par voie de conséquence du recueil entier, elle n'aura pas attendu pour se faire admirer sa publication dans la dernière salve du fabuliste : Le Juge arbitre, le Solitaire et l'Hospitalier a été publiée à peine quelques mois avant, en juin I693, par le P. Bouhours dans un Recueil de vers choisis ${ }^{35}$.

Quelles conclusions, propres à éclairer la disparate du livre ultime des Fables, peut-on tirer de cette esquisse d'un parcours d'invention et de publication dans ces deux genres plus que croisés — torsadés? Celle-ci

35. Le Renard, le Loup et le Cheval, XII, I7, OC I, p. 488-489. Le Milan, le Roi et le Chasseur. À son Altesse sérénissime Monseigneur le prince de Condé, XII, I2, OC I, p. 475-478. Les Compagnons d'Ulysse. À Monseigneur le duc de Bourgogne, XII, I, OC I, p. 45I-454 (le Mercure galant, décembre I690, p. I03-I04). Les deux Chèvres, XII, 4, OC I, p. 459-460 (le Mercure galant, février I69I, p. 237-240). Du Thésauriseur et du Singe, XII, 3, OC I, p. 457-458 (le Mercure galant, mars I69I, p. III-II4). La Ligue des Rats, non recueillie, OC I, p. 543-544 (le Mercure galant, non signée, décembre 1692, p. 24I-244). Le Juge arbitre, le Solitaire et l'Hospitalier, XII, 29, OC I, p. 536-537 (D. Bouhours, S. J., Recueil de vers choisis tirés des meilleurs auteurs [1693], rééd. Paris, J. Barbou, I745, p. 324-326). Notons qu'une version manuscrite qui a tout lieu d'être antérieure à celle-ci se rencontre dans un Recueil de pièces fugitives depuis l'an I660 jusques en I7I4 conservé à la Bibliothèque municipale de Lyon, ms. I67I, p I36-I4O (J. Rougeot, "Une version inédite de la dernière fable de La Fontaine», dans R. Lathuillère (éd.), Mélanges [... F Frédéric Deloffre, Paris, Sedes, 1990, p. 197-212). La composition du texte remonterait à la "conversion» qui suivit la grave alerte de santé qu'avait connue le poète en décembre 1692 (peut-être même avant, selon L. Roche, La Vie de Jean de La Fontaine, Paris, Plon, 1913, p. 364). 
d'abord : par rapport à notre manière actuelle d'envisager la publication d'une œuvre poétique comme un fait ponctuel, à une date précise, sous une forme unique et définitive, la pratique que révèle cet aperçu, si lacunaire soit-il, décompose l'acte de publication entendue au sens large de soumission d'un texte à un public en trois étapes sinon toujours avérées, en tout cas virtuellement enchaînées : la circulation de la pièce encore autonome, puis son regroupement dans un recueil composite de pièces diverses par le genre et l'époque, voire par l'auteur, enfin son insertion dans un recueil à unité générique. La composition bigarrée du douzième livre des Fables semble avoir procédé d'une contamination de modèles : exactement, de la projection du modèle second, celui du recueil composite, sur le dernier, celui du recueil unifié. En dépit de leur dissemblance générique, la proximité esthétique de certaines pièces enrôlées dans les Ouvrages de prose et de poésie des sieurs de Maucroix et de La Fontaine aura pu servir de propédeutique et de justification à leur inscription parmi les fables stricto sensu. Lesquelles de leur côté étaient parvenues à un tel degré de liberté générique dans les livres de I678 et I679 que des modèles voisins semblent être venus concurrencer de manière allusive celui de l'ésopisme originel. Cette liberté pouvait autoriser certains contes à se rencontrer dans un recueil de fables.

À qui verrait là la marque d'une évolution continue, une intention repérable dans l'agencement des livres entre eux apporte le démenti d'une variation plus souple encore : le livre XII était dédié au petit Dauphin, et s'y insérait même une fable composée à sa suggestion et pour son instruction sur le thème du (Vieux) Chat et la (jeune) Souris ${ }^{36}$; le livre entier manifestait d'ailleurs un tour de puérilité retrouvée; on y notait un certain retour aux modèles, aux thèmes et au tour gréco-latins; enfin s'y rencontrait tel conte ou telle nouvelle première manière mêlés à telle idylle ou métamorphose à l'antique, tous bien édifiants. Ces éléments semblent avoir fait hésiter le poète à placer ce livre nouveau en fin de recueil, ou au contraire dans la continuité du premier dédié jadis au grand Dauphin. Ainsi le livre que nous nommons douzième depuis la mort du fabuliste portait-il en titre courant, lors de sa première et seule édition du vivant de La Fontaine, le chiffre VII, comme s'il avait dû suivre les six premiers, tandis que ceux de I678-I679 continuaient de faire bande à part, numérotés de I à V. La publication en volumes séparés autorise ces virements et revirements, imputables peut-être à l'arrivée tardive du Juge arbitre, le

36. Fables, XII, 5, OC I, p. 462. 
Solitaire et l'Hospitalier qui fait une fin si grave et belle au livre qu' elle aura pu lui valoir son rétablissement, au sein du recueil entier, à la place où la chronologie l'appelait.

Quoi qu'il en soit, il appert de cette hésitation que la personne du dédicataire joue un rôle que nous oublions trop aujourd'hui dans l'appréciation et même la composition d'un recueil, où la latitude dans le choix des pièces demeure toujours assez grande. Non sans que d'autres paramètres n'interfèrent, contradictoires parfois. Ainsi, l'édifiante éducation reçue de Fénelon par le duc de Bourgogne n'appelait pas nécessairement le transfert de Belphégor et de La Matrone d'Éphèse dans le recueil qui lui était dédié. Mais c'est toujours la logique du recueil qui aura gouverné le principe de leur présence dans le dernier livre des Fables. Ce procédé éditorial s'inscrit d'ailleurs dans un plus large processus de publication qui parcourt largement le XVII ${ }^{\text {e }}$ siècle et à l'articulation duquel La Fontaine situe sa longue carrière. Héritier des formes de diffusion spécifiques à la poésie durant toute l'époque baroque, il échelonne la divulgation de ses ouvrages par cercles concentriques qui sont autant d'étapes d'un cursus honorum littéraire lui-même à replis et encoches. Suivons-en le parcours raffiné.

La première diffusion d'un poème, c'est la lecture publique dans un cercle amical, mondain ou institutionnel. Diffusion confidentielle encore d'un écrit souvent brouillonné seulement; mais lorsqu'il s'agit de l'Académie française, comme pour la fable Le Renard, le Loup et le Cheval, c'est déjà une apothéose. C'en est une autre lorsque la restriction du cercle des auditeurs s'entend comme une forme d'hommage sélectif au récipiendaire. Ainsi pour l'Adonis offert à Fouquet comme jeton d'entrée à son service. Le surintendant fera superbement calligraphier et enluminer l'exemplaire, à l'instar des grands seigneurs médiévaux composant de manuscrits armoriés et colorés leur bibliothèque privée ${ }^{37}$. Ce qui n'empêche, car rien n'est simple, que l'ouvrage reparaîtra, une décennie plus tard, modifié, imprimé et associé, en compagnie des Amours de Psyché d'abord, puis en I67I dans le recueil de Fables nouvelles et autres poésies qui anticipe, pour les fables, les livraisons de I678-I679 et qui restitue, pour le reste, les pièces inédites de la période Fouquet. Tout se tient, et rien ne se fige. Cette diffusion par lecture élargie en circulation de manuscrits ne laisse de trace que lorsque, par exemple dans la correspondance d'une $\mathrm{M}^{\mathrm{me}}$ de Sévigné, un écho nous en aura été conservé fortuitement. Nous sommes loin, très loin de tout savoir. D'autant que l'anonymat fait partie du contrat, en alternance (sans

37. Copie calligraphiée par N. Jarry, sous mention : «N. Jarry Paris. Scribebat I658», conservé au Musée du Petit Palais. Fac-similé introduit par J. Cordey, Société des bibliophiles français, I93I. 
grande logique) avec la signature chiffrée (M.D.L.F.) et l'aveu oral ou écrit de la pièce livrée au public.

À ce stade toujours, un premier geste de récollection n'est pas interdit : le recueil Du Tralage, le recueil Conrart, celui de l'Arsenal témoignent de cette diffusion et de ce figement temporaire de pièces qui, la plupart manuscrites, peuvent avoir aussi bénéficié de l'impression en plaquette qui leur promet une pérennité précaire. Ce processus culmine dans la publication d'un recueil imprimé dont l'esprit reflète d'ordinaire une disparate modérée par un principe d'unité variable : une génération de poètes, une harmonie de genres et de tonalités, une "école» ou une mouvance tirée par un ou deux maitres éclatants, une thématique et une couleur (satyrique, gaillarde, religieuse...), le goût d'un éditeur peu à peu assimilé au courant poétique dont il a pris coutume de faire converger les flux vers lui. La pratique du recueil poétique au XviI ${ }^{\mathrm{e}}$ siècle ouvre sur un univers d'une complexité et d'une fugacité désarmantes : pratique chiffrée de l'avertissement, stratégie d'ostentation et de discrétion, composition et enchaînements subtilement hiérarchisés, effets d'échos, de connivence ou de répulsion entre les pièces assemblées, et jusqu'à la commande de textes destinés au projet, mettant éventuellement en rivalité leurs auteurs. Toutes choses dans le détail desquelles nous n'entrerons pas ici ${ }^{38}$.

Sauf pour dire que La Fontaine a participé de ces pratiques par plusieurs voies. Il n'a pas fait figure dans les grands recueils de poésie galante enregistrés sous le nom ici d'un éditeur (le recueil dit de Sercy), ailleurs d'un confrère en situation de gouvernement de l'empire poétique (le recueil dit La Suze et Pellisson), ou encore d'une coterie (les Samedis de $\mathrm{M}^{\text {lle }}$ de Scudéry) ${ }^{39}$. Mais il a laissé paraître de gré ou de force trois contes inédits dans le Recueil contenant plusieurs discours libres et moraux et quelques nouvelles en vers non encore imprimées: de gré ou de force, suggérons-nous, car c'est une autre habitude des recueilleurs que de s'emparer des pièces volantes à la mode et de les publier sans l'aveu de leur auteur (à moins que l'auteur ne crie au loup par stratégie!) ${ }^{40}$. Il a d'autre part

38. Voir, après les travaux anciens de F. Lachèvre, A.-M. Garcia, «Les recueils de poésie du début du XvII ${ }^{\mathrm{e}}$ siècle», dans P. Dandrey (éd.), Génétique matérielle, génétique virtuelle. Pour une approche généticienne des textes sas archives, Québec, Les Presses de l'Université Laval, 2009, p. II7-I4I.

39. Poésies choisies de MM. Corneille, Benserade [etc.], Paris, Ch. de Sercy, I653-1660, 5 vol. Recueil de pièces galantes en prose et en vers, de $M^{m e}$ la Comtesse de L[a] S[uze] et de M. Pellisson, Paris, G. Quinet, I664. Madeleine de Scudéry, Paul Pellisson et leurs amis, Chroniques du samedi. Suivies de pièces diverses (1653-I654), éd. critique d'A. Niderst, D. Denis et M. Maître, Paris, Champion, 2002.

40. Voir la note 27 ci-dessus. 
pris en marche et assumé la responsabilité du Recueil de poésies chrétiennes et diverses de $167 \mathrm{I}^{4 \mathrm{I}}$. Il a fabriqué à son usage et en entraînant l'œuvre de son ami Maucroix dans le sillage de sa gloire un recueil consacré à leurs deux œuvres et paru sous leurs deux noms. Il a enfin composé sous son seul nom le recueil à l'instant cité des Fables nouvelles et autres poésies qui joue pour le second recueil des Fables le même rôle que le recueil La Fontaine-Maucroix pour le douzième livre. Sans oublier que les deux contes (La Matrone d'Éphèse et Belphégor) faisant partie de cette livraison ont vu le jour de l'édition, on l'a $\mathrm{dit}^{42}$, dans un recueil d'un autre type encore : dans Le Poème du Quinquina et autres ouvrages en vers, où un inédit de plus ample ambition tire dans son sillage de notoriété attendue des pièces moindres, sans doute déjà divulguées sans avoir jamais encore été recueillies.

Il est notable, de ce point de vue, que faute d'un nouveau recueil de contes et nouvelles, puisqu'il s'était engagé à renoncer au genre, ceux qu'offrait le même recueil La Fontaine-Maucroix (sans compter les idylles et autres imitations de Théocrite ou Ovide) seraient demeurés en recueil composite si le libraire Henry Desbordes ne les avait repris, avec tous ceux qui étaient parus depuis le début de la carrière de La Fontaine, dans une édition des Contes et nouvelles en vers parue à Amsterdam en $1685^{43}$. Ne restaient inédits à sa mort que Les Quiproquos, parus posthumes en I696, et un Conte tiré d'Athénée recueilli par Conrart ${ }^{44}$. Trois fables partagent le même sort, pour des raisons diverses, politiques ou pratiques : Le Renard et l'Écureuil du manuscrit Conrart, écarté (pour être trop politiquement explicite?) du recueil de I668; Le Soleil et les Grenouilles de I672, qu'avait repris avec Le Juge arbitre, le Solitaire et l'Hospitalier le Recueil de vers choisis par Bouhours en I693; La Ligue des Rats de I692, d'attribution plus

4I. Recueil de poésies chrétiennes et diverses. Dédié à Monseigneur le prince de Conti. Par M. de La Fontaine, Paris, P. Le Petit, I67I, 3 vol. On verra l'histoire de la composition de ce recueil et la part qu'y a prise La Fontaine dans OC II, p. 939-946.

42. Voir la note 32 ci-dessus.

43. Contes et nouvelles en vers de M. de La Fontaine, Amsterdam, H. Desbordes, 1685 . La parution prudemment exilée à Amsterdam n'empêche pas la notoriété que signale un compte rendu admiratif de Pierre Bayle dans les Nouvelles de la République des Lettres, en avril I685 (p. 435).

44. Les Quiproquos, dans les Euvres posthumes de La Fontaine réunies par $\mathrm{M}^{\mathrm{me}}$ Ulrich, Paris, G. de Luyne ou J. Pohier, 1996, OC I, p. 919-926.

Conte tiré d'Athénée, indûment enregistré et légèrement remanié sous le titre La Vénus callipyge dans les Euvres choisies de J.-B. Rousseau (Rotterdam, I7I4), avant d'être restitué à La Fontaine en I725 par M. Marais, restitution confirmée par le témoignage du recueil Conrart (Ars. 54I8, t. IX), $O C$ I, p. 927-929. 
douteuse, parue dans le Mercure galant ${ }^{45}$. Cette dernière mention nous donne occasion de saluer enfin un nouveau mode de diffusion de pièces poétiques isolées : dans le cadre d'une publication périodique qui anticipe les revues de poésie des $\mathrm{XIX}^{\mathrm{e}}$ et $\mathrm{Xx}^{\mathrm{e}}$ siècles. Le Mercure fait le relais entre la pratique du recueil composite, d'origine et d'esprit humaniste, et les collections périodiques ou cumulatives de poèmes, de romans et nouvelles, ou de pièces de théâtre qui contribueront grandement, au XVIII ${ }^{\mathrm{e}}$ siècle, à la diffusion des pièces mineures, et parfois majeures aussi, dans ces divers genres.

C'est comme aboutissement et produit de ces processus de publication complexes, fluides et dynamiques qu'il convient de considérer les recueils de fables choisies et de contes et nouvelles en vers qui jalonnent la carrière de La Fontaine. La porosité des deux genres, et plus largement des deux inspirations, trouvait à s'y révéler, les effets d'échos thématiques et formels entre eux à s'y multiplier, et leurs ressemblances à y submerger la conscience généralement maintenue de leur disparité générique. Si la carrière du fabuliste a bien commencé avec Le Meunier, son Fils et l'Âne, qu'un rien suffirait à faire basculer en conte tant le décrochement de la moralité s'y inscrit formellement et structurellement dans le prolongement immanent $\mathrm{du}$ récit à trois épisodes, ne soyons pas surpris, après la malléabilité imprimée aux deux genres par les deux recueils les plus récents, celui des fables de 1678-1679 et celui des Nouveaux contes de 1674, que le livre XII des Fables se soit ouvert à quelques textes de l'obédience adverse, par un effet de la contamination exercée des uns aux autres du fait de leur voisinage dans le recueil La Fontaine-Maucroix de i685. Encore fallait-il peut-être, pour que la barrière de la différence générique fût balayée par le sentiment de la connivence esthétique, une évolution aussi de l'herméneutique poétique dont nous allons, pour terminer, tenter de prendre la mesure.

45. Sur la première, voir ci-dessus, p. 6-7. Et sur La Ligue des Rats, la note 35 ci-dessus. Le Soleil et les Grenouilles traduisait une fable latine du P. Commire parue en feuille volante. Bouhours a accompagné celle de La Fontaine de deux autres versions tournées d'après le même original : une anonyme, peut-être de sa main, et une signée Furetière. Peut-être la fable a-t-elle été écartée du second recueil de I678-1679 pour son actualité trop pointue, peut-être aussi pour la similitude de son titre avec celui de la douzième fable du livre VI. D'après quelques vers des Opera posthuma (I704, p. 2II) du même P. Commire qui remercient La Fontaine d'avoir traduit du latin sa fable L'Âne juge parue en I68I (Poemata, p. 252-253), on attribue parfois à celui-ci un apologue ainsi intitulé et transcrit à la fin d'un Ésope en grec et latin de la bibliothèque de Pontchartrain, portant mention «Par feu mons ${ }^{\mathrm{r}}$ de La Fontaine» (OC I, p. 547-548). Les archaïsmes du style en sont assez suspects pour que l'on penche à rejeter cette attribution. 
Tant que la (petite) fable a calqué, toutes proportions gardées bien sûr, le modèle d'accès au sens qui caractérise la Fable, la grande, tout risque de confusion avec le conte était évité par cette différence de principe. Pour que leur confusion fût possible, il fallait que l'apologue cessât de hanter le ciel de l'allégorie pour se contenter de l'ici-bas de la fiction narrative. Or la carrière de La Fontaine permet de prendre l'exacte mesure de ces enjeux et d'en apprécier la distribution sur pièces et en un lieu et un moment cruciaux où ils concentrent leurs effets : entre les Fables choisies de I668 et les Fables nouvelles de I67I qui ne démentent pas leur qualificatif et promettent déjà la révolution du second recueil; au milieu des trois premières parties des Contes (I665-I666-I67I) et avant que les Nouveaux contes de I674 ne viennent bouleverser là encore la donne et pousser le genre comme au-delà de lui-même et du permis; dans cette période intense de fabrique de contes et de fables, et avant la démarche progressive de renouvellement qu'ils allaient connaître dans la décennie suivante. C'est-à-dire au tout début de 1669. C'est à ce moment précis que Les Amours de Psyché et de Cupidon soldent les comptes de l'inspiration galante de La Fontaine, depuis son lointain Adonis qui avait ouvert les années Fouquet par un récit déjà tiré de la Fable. Or la préface des Amours de Psyché et de Cupidon pose implicitement mais opiniâtrement la question de l'adaptation des récits fabuleux au goût moderne, de leur acclimatation et de leur fonctionnement dans un temps et des lieux où l'on ne croit plus aux dieux, aux monstres ni aux fées : la question du sens que prend un récit de Fable en un temps où la Fable a perdu son sens.

Non moins saturé de mythologie et de féerie que les deux textes qu'on vient de citer, Le Songe de Vaux encore inédit et à jamais inachevé prêtait déjà à l'allégorie, mais une allégorie traitée à distance de rêverie et d'ornementation galantes : en témoignent l'énigme à jamais celée, elle aussi, de la devise "Je suis constant, quoique j'en aime deux", l'écrin mystérieux que le mage Zirzimir avait confié à un druide de jadis, le débat des fées sur la primauté de l'art symbolisé par chacune, l'ekphrasis de l'allégorie du Sommeil peinte par Le Brun, l'évocation des Songes martelant le rêve de Vaux dans l'esprit du dormeur, enfin le personnage d'Aminte dont la signification prête à un badinage du poète sur le déchiffrement de son œuvre :

Le lecteur, si bon lui semble, peut croire que l'Aminte dont j'y parle représente une personne particulière; si bon lui semble, que c'est la beauté des femmes en général; s'il lui plât même, que c'est celle de toutes sortes d'objets. Ces trois explications sont libres. Ceux qui cherchent en tout du mystère, et qui veulent que cette sorte de Poème ait un sens allégorique, ne manqueront pas de recourir aux deux dernières. Quant à 
moi, je ne trouverai pas mauvais qu'on s'imagine que cette Aminte est telle ou telle personne; cela rend la chose plus passionnée, et ne la rend pas moins héroïque ${ }^{46}$.

Délicate moquerie ou aveu indirect d'allégeance, l'énigme et le jeu des clefs étaient passés à ce point dans les mœurs galantes et précieuses que l'on serait tenté de lire ici la projection de l'ambiguïté dans laquelle le processus de déchiffrement de la Fable et, partant, son statut d'écriture allégorique ou narrative se trouvait placé au milieu du XVII siècle.

Adonis prête de même à cette double lecture : partie la plus ancienne du poème, la chasse au monstrueux sanglier qui prive du jour le héros et de son amant terrestre la déesse peut bien passer pour le symbole de la souffrance morbide contenue dans la fleur vénéneuse des amours inégales, selon une herméneutique humaniste qui n'ignore pas les subtilités du décryptage allégorique des ekphraseis dont la traduction de Philostrate par Vigenère avait rappelé sinon renouvelé l'herméneutique ${ }^{47}$. Mais on peut tout aussi bien n'y voir rien d'autre que ce qu'invite à y lire le commentaire tardif dont La Fontaine orne l'édition puis la réédition de la pièce remaniée et associée à Psyché en I669 puis en I671 : un exercice d'écriture héroïque enveloppé dans une idylle à mont et une élégie à val, les trois genres s'unissant par le fil continu d'une narration ornée des images et des figures propres à chacun ${ }^{48}$. Un exercice de style(s), en somme, qui décale la signification symbolique et méditative de la Fable en évocation immanente des nuances sensibles et psychologiques de l'amour heureux et malheureux, sans plus guère de leçon philosophique ni même morale.

C'est le problème que pose de manière beaucoup plus ample et complexe la Fable de Psyché et Cupidon telle que La Fontaine en conte, en varie et en nuance le récit des amours. Car le thème lui est venu tout saturé d'une herméneutique aussi ancienne que complexe : on ne rappellera que pour mémoire l'imprégnation de philosophie platonicienne et de mystique isiaque dont témoigne l'œuvre d'Apulée, puis l'interprétation néoplatonicienne de Plotin qui veut y lire comment l'Âme exilée sur terre s'affranchit par ses tribulations de son incarnation en s'unissant avec

46. Le Songe de Vaux, ouvr. cité, second "Avertissement", OC II, p. 8I. Les passages cités précédemment se situent dans l'Avertissement, p. 79, fragment II, p. 84-96, fragment V, p. IO4-IO6, fragment I, p. 82-83.

47. Adonis, ouvr. cité, OC II, p. IO-I5. Ce déchiffrement allégorique a été très subtilement argumenté par B. Donné dans sa thèse de doctorat de Paris-Sorbonne, La Fontaine et la culture allégorique, 1996. Il y suggère le rapprochement avec le tableau de "La chasse des bestes noires" dans Philostrate, Les Images ou tableaux de platte-peinture. Traduction et commentaire de Blaise de Vigenère, I, 28, éd. critique de F. Graziani, Paris, Champion, 1995, 2 vol., I, p. 396-435.

48. Ouvr. cité, "Avertissement», OC II, p. 3-4. 
Dieu qui est tout Amour, ou l'exégèse chrétienne de Fulgence, appelée à faire école jusqu'au siècle même de La Fontaine, qui traduit le couple de Psyché et de Cupidon comme l'alliance de l'Âme et de la Concupiscence et leurs mésaventures amoureuses comme la déclinaison de cette relation orageuse ${ }^{49}$. Transmise par Boccace à Marino, cette lecture chantournée éclairait encore, quoique d'une lumière indirecte, le récit de la Fable inséré par Marino au chant IV de l'Adone en I623. Après tout, son préfacier, Jean Chapelain, rappelle alors encore que «l'allégorie, dans la commune opinion des bons esprits, fait partie de l'idée du poème, et c'est le second fruit que l'on en peut tirer $^{50} »$. Lequel Chapelain ne manquera pas, en 1656 encore, de proposer un décryptage détaillé, non pas certes philosophique mais pesamment moral, de son poème épique La Pucelle ${ }^{\text {II }}$.

49. "Ils [les conteurs] ont mis une ville pour signifier le monde, où ils ont placé un roi et une reine, comme Dieu et la matière. Ils leur attribuent trois filles, c'est-à-dire la Chair, la Volonté, que nous appelons le Libre Arbitre, et l'Âme. Psyché en effet signifie l'âme en grec; et ils ont voulu qu'elle soit la plus jeune, parce qu'ils voulaient dire que l'âme ne pénètre dans le corps que lorsque celui-ci a déjà été formé; et ils l'ont faite plus belle pour la raison que l'âme est supérieure à la liberté et plus noble que la chair. Vénus lui est hostile, en tant que représentant la Concupiscence; et pour la perdre, elle lui envoie le Désir; mais comme il y a un désir du Mal, il y en a un du Bien : la Concupiscence chérit l'Âme et s'unit à elle; elle tente de lui persuader de ne pas chercher à voir son visage, c'est-à-dire de ne pas apprendre à connaître les délices de la concupiscence — c'est pour cela qu'Adam, bien qu'il soit tout nu, ne se voit pas tel aussi longtemps qu'il n'a pas mangé le fruit de l'arbre de la concupiscence - et aussi de ne pas céder à ses sœurs, c'est-à-dire à la Chair et à la Liberté, qui lui enseignent la curiosité et le désir de voir. Mais Psyché, entrainée par leur contrainte, sort la lampe de sous le boisseau, c'est-à-dire révèle la flamme qui brûle dans sa poitrine et, quand elle l'a vue, la trouve douce et la chérit. On dit qu'elle a brûlé Concupiscence de l'huile de sa lampe parce que toute concupiscence, plus elle est aimée, plus elle est ardente et inflige à la chair la marque du péché. Donc, une fois la concupiscence révélée, elle perd sa grande fortune, court des dangers et est chassée de la demeure royale [etc.]." Traduction ancienne (1734) du texte des Enarrationes allegorica fabularum de Fulgence ( $\mathrm{v}^{\mathrm{e}}-\mathrm{vI}^{\mathrm{e}}$ siècles), citée par Y. Giraud, «Un mythe lafontainien : Psyché» (Studi di leteratura francese, 1990), dans P. Dandrey, La Fontaine/CEuvres galantes, Paris, Klincksieck, coll. «Parcours critique», 1996, p. 190. Texte original des Enarrationes: éd. de Milan, V. Scinzeneler, I498, in-f ${ }^{\circ}$, III, 6.

50. Cité par B. Beugnot, «Pour une poétique de l'allégorie classique», dans M. Fumaroli (éd.), Critique et création littéraires en France au XVII siècle (Colloque du CNRS, Paris, 4-6 juin 1974), Paris, Éditions du CNRS, 1977, p. 413.

5I. "Je disposai toute sa matière de telle sorte que la France devait représenter l'âme de l'homme en guerre avec elle-même, et travaillée par les plus violentes de toutes les émotions; le roi Charles: la volonté, maîtresse absolue, et portée au bien par sa nature, mais facile à porter au mal, sous l'apparence du bien; l'Anglais et le Bourguignon, sujets et ennemis de Charles : les divers transports de l'appétit irascible qui altèrent l'empire légitime de la volonté; Amauri et Agnès, l'un favori et l'autre amante du prince : les différents mouvements de l'appétit concupiscible qui corrompent l'innocence de la volonté, par leurs inductions et par leurs charmes [etc.].» (J. Chapelain, La Pucelle ou la France délivrée, poème hérö̈que en douze chants, Paris, A. Courbé, 1656, éd. critique d'É. de Molènes, Paris, Flammarion, coll. "Collection des épopées nationales», s. d., 2 vol., I, p. LVIII.) 
Ces hautes ambitions ne rendent que plus piquante la déréliction, certes polémique, que leur oppose Perrault pour débouter de leurs prétentions les partisans des Anciens au profit des Modernes et des contes modernes :

À l'égard de la morale cachée dans la fable de Psyché, fable en elle-même très agréable et très ingénieuse, je la comparerai avec celle de Peau d'Âne quand je la saurai, mais jusqu'ici je n’ai pu la deviner. Je sais bien que Psyché signifie l'âme; mais je ne comprends point ce qu'il faut entendre par l'Amour qui est amoureux de Psyché, c'est-àdire de l'Âme, et encore moins ce qu'on ajoute, que Psyché devait être heureuse, tant qu'elle ne connaitrait point celui dont elle était aimée, qui était l'Amour, mais qu'elle serait très malheureuse dès le moment qu'elle viendrait à le connaître : voilà pour moi une énigme impénétrable. Tout ce qu'on peut dire, c'est que cette Fable de même que la plupart de celles qui nous restent des Anciens n'ont été faites que pour plaire sans égards aux bonnes mœurs qu'ils négligeaient beaucoup ${ }^{52}$.

L'agrément et l'ingéniosité à quoi se ramène tout l'intérêt de Psyché, ce pourrait bien être aussi l'approche de La Fontaine si l'on en juge par sa préface à l'ouvrage toute obsédée de la difficulté de forme que lui a donné la menée de ce récit antique accommodé au goût du siècle. Et lorsqu'il donne pour double motif de son ouvrage "qu'il plût, et que même on y trouvât du solide aussi bien que de l'agréable ${ }^{53}$ ", il est notable que le solide se cantonne pour lui à l'ornementation poétique du récit par le prosimètre, à l'évocation en style soutenu des enfers où descend Psyché, à la description de Versailles, contrepoint "réaliste" aux fantasmagories du palais de Cupidon, et aux réflexions poéticiennes des quatre amis s'y promenant et devisant sur l'effet esthétique de l'ouvrage que l'un d'eux conte aux trois autres. Rien de moral, rien de philosophique, rien de symbolique, rien de métaphysique. Point de vue tout "formaliste», dirions-nous aujourd'hui.

C'est qu'à vrai dire l'allégorie a fort à faire avec une ennemie qu'elle loge en son sein : l'image, dont le chiffre doit lui permettre de signifier par détour et d'illuminer par une lumière à la fois intérieure et supérieure. Mais qu'arrive-t-il lorsque le poète, comme c'est le cas de La Fontaine, cède à l'enchantement de l'image, à l'enchantement dans l'image? Dès lors, le phénomène s'inverse : la volupté que sécrète la représentation figurée fait sourdre de toute réalité transmuée en image une jouissance des sens et de l'esprit qui constitue peut-être le plus haut sens accessible à l'humaine nature. Conception moins abstraite et intellectuelle que celle

52. Ch. Perrault, Préface des Contes en vers (Grisélidis, nouvelle, avec le Conte de Peau d'Âne et celui des Souhaits ridicules, Paris, J.-B. Coignard, $4^{\mathrm{e}}$ éd., I695) réunis aux Histoires ou Contes du temps passé (I697), éd. critique de R. Zuber, Paris, Imprimerie nationale, «Lettres françaises», I987, p. $8 \mathrm{I}-82$.

53. Préface des Amours de Psyché et de Cupidon, OC II, p. I26. 
de l'idéalisme allégorique : le plaisir des sens fait ici part égale avec l'esprit dans la délivrance du Sens. Dans Psyché, comme auparavant dans Adonis qui lui avait ouvert la voie, et dans Le Songe de Vaux qui l'avait à son tour empruntée, les scènes d'amour et plus généralement de sensualité sont gouvernées par une esthétique de l'image peinte, de l'ekphrasis enchantée et fascinante, sans autre finalité que sa propre saveur. L'œil semble invité à en épouser la délicate représentation plutôt qu'à en perforer la toile pour en extraire une signification latente : il n'est que de voir Adonis "près des bords d'un ruisseau, couché sur des gazons, rêv[ant] au bruit de l'eau ${ }^{54}$ ». Ne croirait-on pas Narcisse peint par Poussin? Puis c'est la Nuit de Le Brun tout ensommeillée au plafond d'un salon de Vaux; et c'est encore Aminte assoupie sous un arbre du parc; enfin et par-dessus tout, c'est le spectacle célèbre et si souvent représenté de Cupidon dormant :

Il dormait à la manière d'un dieu, c'est-à-dire profondément, penché nonchalamment sur un oreiller, un bras sur sa tête, l'autre bras tombant sur les bords du lit, couvert à demi d'un voile de gazess.

Démarquée d'une image — dessin, gravure ou tableau — qui féconde l'imagination sensuelle du poète, la description avoue presque cet artifice originel pour mieux susciter l'émotion : celle qui nous saisirait à la vue d'une figure peinte saisie par la vie, d'une sculpture qui respirerait. Détour sans doute, mais détour autre que celui de l'allégorie : détour par l'artifice qui restitue à sa volupté le spectacle de la réalité. Comment ne pas y voir l'expression d'une confiance dans la fiction pour restituer la réalité à son principe de délectation — anticipation de l'hymne à la Voluptés ${ }^{5}$ qui, démarqué de Lucrèce, ne procède pas tant de la philosophie épicurienne que d'une poétique de l'éloge, bien avant Saint-John Perse?

Ainsi l'esthétique de l'enchantement par l'image, perturbant le mécanisme attendu du décryptage allégorique, de la quête du sens par-delà les apparences séductrices de la fiction, induit-elle une forme toute particulière d'accès à la signification de l'œuvre littéraire. Modelée sur l'analyse des effets de l'ekphrasis, la "révélation» attendue de la poésie prend la forme d'une participation voluptueuse à la réalité par le détour de l'image constitutive des récits de la Fable. La délectation procurée par la lecture de ces textes offre un exemple et suggère un apprentissage de cet hédonisme dont le songe fournit un équivalent assez exact : puissance de la

54. Adonis, OC II, p. 6.

55. Les Amours de Psyché et de Cupidon, OC II, p. I73.

56. Ibid., p. 257-258. 
sensation, intimité de sa manifestation, il ne manque au songe que d'être conscient et maitrisable pour figurer parfaitement cette sagesse de l'enchantement contrôlé. La fiction littéraire y pourvoit, qui édifie comme les zélés serviteurs du dieu Sommeil dans Le Songe de Vaux des palais de rêverie à volonté, des temples dédiés à une Volupté apprivoisée et docile, qui participe à l'édification esthétique du moi. Le régime d'émergence du sens dans les poèmes dérivés de la "grande» Fable, mythologique ou féerique, calque donc en l'occurrence le modèle des "petites", le modèle des apologues ésopiques revêtus par La Fontaine de la livrée des Muses ${ }^{57}$. Le "gai savoir " que l'apologue suggère pour sagesse universelle par-delà les moralités explicites et disparates propres à chaque récit; cette sagesse souriante, distancée et voluptueuse qui procède de l'expérience esthétique du charme lucide, du songe vigilant induit par le décalage animalier, les ouvrages galants la professent aussi : elle revêt l'aspect d'une esthétique et d'une éthique de la volupté, effet d'une transmutation du monde en objet de délectation, à la faveur du décalage mythologique, de la forme onirique et du ton de dérision modérée qui caractérisent la mise en œuvre poétique de ces récits.

Adieu à l'allégorisme? Pas tout à fait. Entre l'orthodoxie allégorique de Fulgence et le radicalisme iconoclaste de Perrault, Chapelain avait suggéré une posture intermédiaire, et l'avait fait en l'appuyant sur l'exemple hautement significatif pour nous des apologues ésopiques :

L'allégorie donc de la commune opinion des bons esprits fait partie de l'idée du poème et est le second fruit que l'on peut en retirer. Or, comme il arrive qu'elle soit le plus souvent incompatible avec le véritable succès des choses, les poètes obligés à l'y faire entrer se résoudront toujours plutôt à fausser la vérité laquelle n'est en leurs ouvrages que par accident qu'à laisser l'allégorie, qui y doit être par nature. De quoi nous avons une notable preuve dans les fables qu'Ésope a données à son pays. Ontelles aucune vraisemblance, non pas seulement vérité, pour ce qui est des arraisonnements, paroles, subtilités, prévoyances et autres choses qu'il attribue à ses animaux? et néanmoins elles ont passé jusqu à nous avec un applaudissement général du monde, qui lisant la fable va soudain à son sens, c'est-à-dire à l'autre espèce désignée, appliquant utilement ce qu'il a dit d'une impossible à une possible, sans s'amuser à en examiner la possibilité, comme pour nous avertir plus clairement quaux autres fables

57. Sur la légitimité du parallèle entre «grande» et «petite» fable, citons le P. Le Bossu : «Pour mieux faire voir qu'une épopée est une véritable fable, et que ce terme qu'on lui applique, n’est point en cela métaphorique et figuré, mais qu'il est son sens propre et naturel; et que ce sens est le même que quand on donne le nom de Fables aux fictions d'Ésope : je vais mettre ici en parallèle la Fable de l'Iliade et celle d'Ésope.» (Traité du poème épique, p. 51.) Voir A. Gaillard, Fables, mythes, contes - l'esthétique de la fable et du fabuleux (I660-I724), Paris, Champion, coll. "Lumière classique", première partie, chap. I et II. 
(j'entends poésies ordonnées et plus proches de nous que celles-là) laissant l'examen de la vérité comme chose indifférente, il importe seulement de regarder si le profit recherché s'y rencontre ${ }^{58}$.

La "petite» fable vient ici au secours d'une évaluation de la grande — de la Fable. Pour Chapelain, toute œuvre d'art est par nature allégorique. Si bien que l'invraisemblance de la fiction nécessaire à en esquisser le profitable enseignement est légitimée par la vérité au service de laquelle est enrôlée son imagerie parfois incroyable. Ainsi des fables d'Ésope, invraisemblables mais crédibles, car véritables. La Fontaine n'eût pas contredit à cette analyse. Nous avons tenté, naguère, de montrer comment il approfondit et renouvela la vérité morale des Fables, en faisant jaillir de leur invraisemblance une énergie et un effet de réalité supérieurs à ceux d'une fiction plus crédible ${ }^{59}$. Au sein de l'écart, du "jeu» un peu schématique entre la vérité de fond et l'invraisemblance d'aspect qui caractérisait l'apologue ésopique, la fable lafontainienne dégage désormais sa signification, pour partie inédite, de l'effet de réalité produit, paradoxe de l'image, par les hybrides mi-humains mi-animaux, ni humains ni animaux, qui en peuplent la scène onirique. Le décalage induit par ces mufles parlants confère aux comportements et aux sentiments qu'ils figurent, à la faveur de ce détour par une improbable "réalité» digne seulement du rêve ou du cauchemar, une véracité incisive et révélatrice comme celle des songes, celle des images, celle de l'imagination. Enveloppant les préceptes naifs et souvent contradictoires de la sagesse des nations récitée par le bon Ésope, une vision morale nouvelle, moins prescriptive que descriptive, se déduit de cette traverse par la fiction, de cette échappée rêveuse.

Quel en est le propos et quelle en est l'assise? Oscillant entre l'acuité de l'observation et la dérive enchanteresse du songe, cette philosophie poétique épanouie au cour d'une délectation par les images d'un rêve éveillé propose pour leçon la plus utile à l'humaine condition une sagesse de la délectation : cette délectation même que procure le songe au rêveur vigilant, capable de contempler, de connaître et de conjurer dans la distance apaisée de ces fictions instructives le cauchemar de la réalité. Songe vigilant, la fable déduit sa sagesse morale du parti esthétique requis par son récit. Le régime allégorique de la signification qui œuvrait dans l'apologue ésopique a laissé place ici au processus inédit d'une émergence du sens

58. J. Chapelain, préface de l'Adone, éd. critique de G. Pozzi, Milan, Adelphi, I988, 2 vol., I, p. $25-26$.

59. Dans La Fabrique des fables. Poétique de La Fontaine, Paris, Klincksieck, (1991), I992², et PUF, coll. «Quadrige», 1996. 
au cœur et à la faveur de l'infusion délectable des images dans l'âme songeuse : la transcendance orgueilleuse s'est rabattue sur une immanence hédoniste. Remplaçons l'âme songeuse par l'esprit rieur et la sensualité chatouillée, et les contes deviennent justiciables du même décryptage. L'évolution du premier au second recueil des Fables, l'évolution du premier au dernier recueil des Contes disent cette autre évolution, réciproque et combinée, de chacun des deux genres vers l'autre : sans entamer le socle de leur définition structurale et sans pulvériser la dissemblance constitutive qui les identifie, le glissement de la sagesse des fables en une philosophie de la délectation auquel l'enchantement du récit initie les âmes, et le glissement de la pure jouissance du récit des contes en une (anti-)sagesse de la délectation sensuelle appelaient sinon leurs itinéraires à se croiser, du moins leurs ondes à interférer au cercle le plus large de leur propagation. Contaminé par le modèle du recueil composite dont la diversité participe à la saveur, le XII livre des Fables aura discrètement accompli, sinon en la signant tout à fait, du moins en la signalant par l'exemple, cette évolution vers une osmose partielle. Les Amours de Psyché et de Cupidon, dans le prolongement des pièces du temps de Vaux, auront présidé à cette évolution qui est une révolution.

Nous conclurons en nous interrogeant sur ce qui s'est joué dans cette évolution dont nous venons de soutenir l'hypothèse, à partir d'un indice qui sera peut-être jugé trop mince pour avoir mobilisé tant d'examen. Renchérissons donc sur le caractère plus encore hypothétique de ce que nous allons en tirer. Car encore faut-il généraliser parfois pour comprendre. Nous n'avons pas posé tout de go ni sans nuance le postulat d'une occultation de l'herméneutique allégorique dans les Lettres et plus généralement les arts au cours du XVII ${ }^{\mathrm{e}}$ siècle : c'est-à-dire entre l'âge de l'Humanisme, qui a promu le concept de Belles et Bonnes Lettres à l'autonomie d'une laïcité implicite, et le siècle des Lumières, qui va promouvoir l'autonomie de l'esthétique rejaillissant en une définition totalement bouleversée de l'empire des Belles ou des Bonnes Lettres sous le vernis d'une poétique apparemment inchangée. Nous suggérons seulement que le mode d'accès au sens de la fiction narrative a durant le Grand Siècle parachevé sa révolution humaniste et commencé sourdement son évolution vers de nouvelles lumières. Qu'est-ce à dire dans l'ordre des contes et des fables? Ceci : que la relégation de leur différence générique, fondée sur la différence de leur rapport à l'herméneutique allégorique, et la promotion de leur similitude dans l'ordre du récit de fiction joueuse et joyeuse induisant sa leçon dans l'immanence de la fruition délectable qu'en produit la narration, 
signalent un infime déplacement, mais déterminant. Ce déplacement, qui consiste à concevoir que le sens de l'œuvre d'écriture fictive travaillée en poème se condense dans la projection de sa forme sur sa portée, équivaut à lui reconnaître pour principe de sa définition sa littérarité. Si fables et contes, chez La Fontaine, se superposent même partiellement, c'est en tant qu'œuvres littéraires conscientes de leur spécificité; des œuvres où l'instruire, loin de se résorber dans le plaire, s'en nourrit, s'y féconde, s'y distille, s'y consomme sans reste ni rebut. Appeler littéraires les ouvrages de Belles Lettres de jadis, c'est leur reconnaître pour principe, évidemment, que le Beau y soit la splendeur du Vrai, sans perte ni résidu. Autrement dit qu'elles relèvent de l'esthétique. Si le Xvir ${ }^{\mathrm{e}}$ siècle fut bien le lieu de ce passage, la relation entre fables et contes en accuse à sa façon le mouvement par la substitution d'une intuition de semblance fondée sur leur communauté narrative en lieu et place de la conscience de leur dissemblance fondée sur leur divergence générique - cette substitution ne pouvant opérer qu'à la faveur d'une révolution dans le processus d'accès au sens qu'on leur prête, une révolution herméneutique non tant en défaveur qu’au sein même du chiffre et du déchiffrement allégorique.

Pour mesure, expression et preuve de cette révolution herméneutique, resterait à avouer d'où est sorti tout ce que dessus, toute la réflexion qu'on vient d'essayer. D’un essai de Montaigne, bien sûr car, sinon tout, la plupart du meilleur au XVII ${ }^{\mathrm{e}}$ siècle en est issu et tissu. Voici, qui se tient en un endroit névralgique de l'ouvrage - sa presque fin des fins :

Ésope, ce grand homme, vit son maître qui pissait en se promenant : "Quoi donc, fit-il, nous faudra-t-il chier en courant?» Ménageons le temps; encore nous en restet-il beaucoup d'oisif et mal employé. Notre esprit n'a volontiers pas assez d'autres heures à faire ses besognes, sans se désassocier du corps en ce peu d'espace qu'il lui faut pour sa nécessité. Ils veulent se mettre hors d'eux et échapper à l'homme. C'est folie; au lieu de se transformer en anges, ils se transforment en bêtes; au lieu de se hausser, ils s'abattent. Ces humeurs transcendantes m'effrayent, comme les lieux hautains et inaccessibles; et rien ne m'est à digérer fâcheux en la vie de Socrate que ses extases et ses démoneries, rien si humain en Platon que ce pour quoi ils disent qu'on l'appelle divin ${ }^{60}$.

Le frémissement à peine perceptible des Fables et des Contes en direction de leur coalescence, peut-être participe-t-il, depuis sa place infime, à cette mise à mort du démon de Socrate dont allait naître la littérature.

60. Les Essais, III, XIII, «De l’Expérience», P. Villey (éd.) [1924], rééd. et préf. par V.-L. Saulnier, PUF, I965 [I988], p. III5. 
Qu'Ésope y soit convié, et comme héros d'un conte — à peine : d'une anecdote, d'un mot - dont l'allégorie du plus haut sens se rabat sur la truculence du bas corporel pour en débusquer les feintises de la transcendance dans la jubilation d'un éclat de rire qui éclate de lumière immédiate à l'esprit, cela ne boucle-t-il pas le cercle? 